\title{
Covariance Operators of Skew Distributions
}

\author{
By \\ Yoshiomi NAKAGAMI*
}

In this paper we extend the concept of a skew distribution on a real Hilbert space $H$ defined in [4] and [6] to that on a complex Hilbert space $K$ with an antiunitary involution $\Gamma$, and show the following result which stems from Theorem 3 in [4]. Whenever a skew distribution $m$ is given on even or infinite dimensional $(K, I)$, any two of the following conditions imply the other one:

(i) $m$ is a factor distribution;

(ii) $m$ is $\mathscr{U}$-invariant; and

(iii) any pair of $\Gamma$-invariant orthogonal subspaces are independent with respect to $m$.

In the appendix we give a correspondence of a pair of Fock and anti-Fock representations to a pair of orthogonal transformations $\left\{\Lambda,{ }^{t} \Lambda\right\}$ with $\Lambda^{2}=-1$ on $H$.

\section{Notations and Definitions}

In this section we prepare some notations and definitions from papers $[2],[4]$ and $[6]$ with slight modifications.

Let $\mathscr{C}$ be a separable complex Hilbert space, $\mathscr{U}$ a von Neumann algebra on $\mathscr{C}$ and $E$ a faithful normal trace on $\mathfrak{U}$ with $E(1)=1$. By $(K, \Gamma)$ we mean a complex Hilbert space $K$ with an antiunitary involution $\Gamma$, namely, $(\Gamma \xi \mid \Gamma \eta)=(\eta \mid \xi)$ for $\xi, \eta \in K$ and $\Gamma^{2}=1$. We denote by $F=(F$, श) a strongly continuous $(\|F(\xi)\| \leqq \lambda\|\xi\|$ for some $\lambda>0$ ) and faithful (if $\xi$

Received November 10, 1970.

Communicated by H. Araki.

* Department of Mathematics, Tokyo Institute of Technology, Ookayama, Meguro-ku, Tokyo, Japan. 


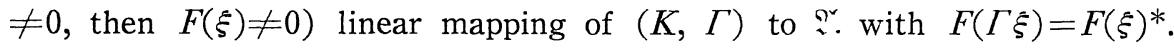
Since $E$ is faithful, $F \neq 0$. For any subset $K_{0}$ of $K$ the von Neumann algebra generated by $F(\xi), \xi \in K_{0}$ is denoted by $\mathfrak{Y}:\left(K_{0}\right)$. $\stackrel{Y}{\mathfrak{Y}}(F)$ is the union of $\mathfrak{A}\left(K_{0}\right)$ where $K_{0}$ runs over all finite subsets of $K$. Introduce the following equivalence relation into the set $\mathbf{F}$ of such linear mappings. $\left(F_{1}\right.$, $\left.\mathfrak{I}_{1}\right)$ and $\left(F_{2}, \mathfrak{A}_{2}\right) \in \mathbf{F}$ are equivalent if for any finite subset $K_{0}=\left\{\xi_{1}, \ldots\right.$, $\left.\xi_{n}\right\}$ of $K$ there exists an isomorphism $\tau: F_{1}(\xi) \rightarrow F_{2}(\xi), \xi \in K_{0}$ of ${ }^{\mathfrak{r}_{1}}\left(K_{0}\right)$ onto $\mathfrak{A}_{2}\left(K_{0}\right)$ such that

$$
E_{1}(A)=E_{2}(\tau(A))
$$

for $A \in \mathfrak{A}_{1}\left(K_{0}\right)$, where $E_{i}$ is a faithful normal trace on $\mathfrak{U}_{i}$ with $E_{i}(1)=1$.

An equivalence class $m$ of $\mathbf{F}$ classified by the above relation is called a distribution on $(K, \Gamma)$. Since algebraic structures of all $(F, \mathfrak{2} \cdot) \in m$ is preserved by an isomorphism as above, the terminologies for $m$ is utilized as similarly as that for each $(F, \mathfrak{A})$. For $(F, \mathfrak{2}) \in m$, let $\mathfrak{S}_{F}, \pi_{F}$ and $\Omega_{F}$ be the Hilbert space, the representation and the cyclic unit vector respectively such that

$$
E(A)=\left(\pi_{F}(A) \Omega_{F} \mid \Omega_{F}\right)
$$

for $A \in \mathfrak{H} .(F)$. Denote

$$
\pi_{F}(\mathfrak{X} \cdot)=\left\{\pi_{F}(A): A \in \mathfrak{Y} \cdot(F)\right\}^{\prime \prime} .
$$

Then it is easily seen that $(F, \mathfrak{N})$ and $\left(\pi_{F} \circ F, \pi_{F}(\mathfrak{U})\right)$ are equivalent. The latter is called the standard representative of $m$. From this we may assume that for every $(F, \mathfrak{2}$. $) \in m$,

$$
\text { I. }=\{F(\xi): \xi \in K\}^{\prime \prime}
$$

in the following. If $\mathfrak{Q}$ is a factor for $(F, \mathfrak{2}) \in m, m$ is called a factor distribution.

Let $K_{1}$ be a $\Gamma$-invariant subspace of $K$ such that $\Gamma_{1}=\Gamma \mid K_{1}$. For distributions $m$ on $(K, \Gamma)$ and $m_{1}$ on $\left(K_{1}, \Gamma_{1}\right)$ choose suitable representatives $(F, \mathfrak{R}) \in m$ and $\left(F_{1}, \mathfrak{A}_{1}\right) \in m_{1}$. If $\mathfrak{P}_{1} \subset \mathfrak{Y}$. and $F(\xi)=F_{1}(\xi)$ for $\xi \in K_{1}$, then $m$ is called an extension of $m_{1}$ and denoted by $m_{1}=m \mid K_{1}$.

Since for any distribution $m$ on $(K, \Gamma)$ there is $\lambda>0$ such that 


$$
E\left(F(\xi)^{*} F(\xi)\right) \leqq \lambda \|\left.\xi\right|_{1} ^{2}
$$

for all $\xi \in K$, there exists a positive operator $t$ on $K$ such that

$$
E(F(\eta) * F(\xi))=(t \xi \mid \eta)
$$

which is called the covariance operator of $m$. It is easily seen that $t=\Gamma t \Gamma$.

A family $\left\{K_{\iota}: \iota \in I\right\}$ of subspaces of $K$ is said to be independent with respect to $m$, if

$$
E\left(A_{\iota_{1}} \ldots A_{\iota_{n}}\right)=E\left(A_{\iota_{1}}\right) \ldots E\left(A_{\iota_{n}}\right)
$$

for any $A_{\iota} \in \mathfrak{Y} \cdot\left(F_{\iota}\right)$ with $F_{\iota} \in m \mid K_{\iota}$ and for any $\iota_{1}, \ldots, \iota_{n} \in I$.

A distribution is skew, if for any $(F, \mathfrak{Q}$. $) \in m$

$$
[E(\xi), F(\eta)]_{+} \in \mathfrak{Y} \cap \cap \mathfrak{X}^{\prime}
$$

and if $F \in m$ implies $-F \in m$. It follows from the last condition that

$$
E\left(F\left(\xi_{1}\right) \ldots F\left(\xi_{2 n+1}\right)\right)=0 .
$$

A unitary operator on $(K, \Gamma)$ which commutes with $\Gamma$ is called a Bogoliubov transformation. $\mathscr{U}$ is a set of Bogoliubov transformations on $(K, \Gamma)$ whose commutant is the algebra of scalar operators. A skew distribution $m$ is called to be $\mathscr{U}$-invariant if $(F, \mathfrak{U}) \in m$ implies $(F \circ U, \mathscr{U})$ $\in m$ and if

$$
[F(U \xi), F(U \eta)]_{+}=[F(\xi), F(\eta)]_{+},
$$

here $(F \circ U)(\xi)=F(U \xi)$.

A self dual CAR algebra $\mathfrak{V}_{S D C}(K, \Gamma)$ over $(K, \Gamma)$ is a *algebra generated by $B(\xi), \xi \in K$, its adjoint $B(\xi)^{*}, \xi \in K$ and the identity which satisfy the following three relation: $B(\xi)$ is linear in $\xi,[B(\xi), B(\eta)]_{+}=$ $(\xi \mid \Gamma \eta) 1$ and $B(\xi)^{*}=B(\Gamma \xi)$. If $K$ has a finite dimension, $\mathfrak{A}_{S D C}(K, \Gamma)$ has a finite dimension. Irrespective of the dimension of $K, \mathfrak{X}_{S D C}(K, \Gamma)$ has a unique $C^{*}$-norm and $\overline{\mathfrak{U}}_{S D C}(K, \Gamma)$ denotes its $C^{*}$-completion.

A state $\varphi$ on $\overline{\mathfrak{V}}_{S D C}(K, \Gamma)$ satisfying the following relation is called a quasifree state:

$$
\varphi\left(B\left(\xi_{1}\right) \ldots B\left(\xi_{2 n+1}\right)\right)=0
$$




$$
\varphi\left(B\left(\xi_{1}\right) \ldots B\left(\xi_{2 n}\right)\right)=\sum \operatorname{sgn}(s) \prod_{j=1}^{n} \varphi\left(B\left(\xi_{s(2 j-1)}\right) B\left(\xi_{s(2 j)}\right)\right),
$$

where $n=1,2, \ldots$, the sum is over all permutations $s$ satisfying

$$
s(1)<s(3)<\cdots<s(2 n-1) \text { and } s(2 j-1)<s(2 j)
$$

for $j=1, \ldots, n$ and $\operatorname{sgn}(s)$ is the signature of $s$.

\section{Results}

Lemma 1. Let $t$ be a bounded positive operator. If $(t \xi \mid \eta)=0$ for any pair of $\xi$ and $\eta$ in $K$ with $(\xi \mid \eta)=(\xi \mid \Gamma \eta)=0$, then $t$ is a scalar operator.

Proof. Choose a complete orthonormal system $\left\{\xi_{\iota}: \iota \in I\right\}$ of $K$ with $\xi_{\iota}=\Gamma \xi_{\iota}$. It follows from the hypothesis that $\left(t \xi_{\iota} \mid \xi_{\kappa}\right)=0$ for $\iota, \kappa \in I$ with $\iota \neq \kappa$, and hence $t \xi_{\iota}=\lambda_{\iota} \xi_{\iota}$ for some $\lambda_{\iota} \geqq 0, \iota \in I$. For any $\iota_{0}$ and $\iota_{1}$ in $I$ with $\iota_{0} \neq \iota_{1}$, put

$$
\eta_{\iota_{0}}=\xi_{\iota_{0}}-\xi_{\iota_{1}}, \eta_{\iota_{1}}=\xi_{\iota_{0}}+\xi_{\iota_{1}}
$$

and $\eta_{\iota}=\xi_{\iota}$, for $\iota \neq c_{0}$ and $\iota \neq c_{1}$. Then $\Gamma \eta_{\imath}=\eta_{\iota}$ for $\iota \in I$ and $\left\{\eta_{\imath}: \iota \in I\right\}$ is a complete orthogonal system. It follows from the hypothesis that $t \eta_{t_{0}}$ $=\mu \eta_{t_{0}}$ for some $\mu \geqq 0$. On the other hand

$$
t \eta_{\iota_{0}}=t\left(\xi_{\iota_{0}}-\xi_{\iota_{1}}\right)=\lambda_{\iota_{0}} \xi_{\iota_{0}}-\lambda_{\iota_{1}} \xi_{\iota_{1}} .
$$

Thus $\lambda_{\iota_{0}}=\mu=\lambda_{\iota_{1}}$. Repeating the similar argument for each pair of elements in $I$, we get $\lambda_{\iota}=\mu$ for $\iota \in I$. Consequently $t=\mu 1$.

Corollary. Let $t$ be the covariance operator of a skew distribution $m$ on $(K, \Gamma)$. If $[m(\xi), m(\eta)]_{+}=0$ for any pair of $\xi$ and $\eta$ in $K$ with $(\xi \mid \eta)=(\xi \mid \Gamma \eta)=0$, then $t$ is a scalar operator.

Proof. It is clear from

$$
2(t \xi \mid \eta)=E\left(\left[F(\xi), F(\eta)^{*}\right]_{+}\right)
$$

for $(F, \mathfrak{A}) \in m$.

It should be noted that, since the underlying Hilbert space $\mathfrak{S}$ of $\mathfrak{X}$. for $(F, \mathfrak{2}$. $) \in m$ is assumed to be separable, $\mathscr{N}(F)^{\prime}$ is generated by a countable family of elements. Furthermore, since $F$ is assumed to be faithful, it follows that $K$ is separable. 
Lemma 2. A skcw distribution $m$ with the covariance operator $t$ has a represcntative $(F, \stackrel{\because}{\therefore}) \in m$ such that there exist a locally compact space $Z$, a positive measure $\nu$ whose carrier is $Z$, a $\nu$-measurable field $\zeta \rightarrow \varsubsetneqq(\zeta)$

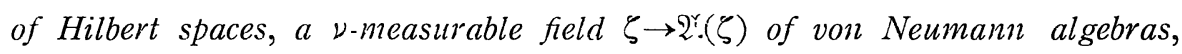

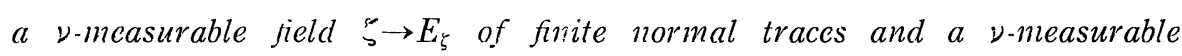
operator valued function $\zeta \rightarrow t(\zeta)$ with $t(\zeta) \in \mathbf{B}(K)$ and that

$$
\begin{aligned}
& \curvearrowleft=\int^{\oplus}(\zeta) d \nu(\zeta), \stackrel{\Upsilon}{\Upsilon}=\int^{\oplus} \stackrel{\Upsilon}{\Upsilon}(\zeta) d \nu(\zeta), E=\int^{\oplus} E_{\zeta} d \nu(\zeta), \\
& {\left[F(\xi), F(\eta)^{*}\right]_{-}=2 \int^{\oplus}(t(\zeta) \xi \mid \eta) 1(\zeta) d \nu(\zeta)}
\end{aligned}
$$

and

$$
t=\int t(\zeta) E_{\zeta}(1(\zeta)) d \nu(\zeta)
$$

where $\mathbf{B}(K)$ is a full opcrator algebra on $K$ and $1(\zeta)$ is the identity in $\mathfrak{2}(\zeta)$.

Proof. According to the reduction theory and the separability of we can conclude that there exist a locally compact space $Z$ which satisfies the second axiom of countability, a positive measure $\nu$ whose carrier is $Z$, a $\nu$-measurable field $\zeta \rightarrow \mathfrak{C}(\zeta)$ of non zero Hilbert spaces on $Z$ and a $\nu$ measurable field $\zeta \rightarrow \mathfrak{U}(\zeta)$ of factor von Neumann algebras over $\mathfrak{(}(\zeta)$ on $Z$ such that or is spatially isomorphic to

$$
\int^{\oplus} \mathfrak{A}(\zeta) d \nu(\zeta) \text { over } \int_{\mathscr{C}}^{\oplus}(\zeta) d \nu(\zeta)
$$

Since $K$ is separable, it contains a $\Gamma$-invariant countable dense $\mathbf{Q}$ linear subset $K_{0}$ where $\mathbf{Q}$ denotes the field of rational complex numbers. Since $F$ is strongly continuous, a ${ }^{*}$-algebra $\mathfrak{2}_{0}$ generated by $\{F(\xi): \xi \in K\}$ and the identity has a countable base with respect to the uniform topology. Hence we may associate with $T \in \mathfrak{X}_{0}$ a $\nu$-measurable field $\zeta \rightarrow T(\zeta)$ of operators with $T(\zeta) \in \mathfrak{A}(\zeta)$ such that

$$
T=\int^{\oplus} T(\zeta) d \nu(\zeta),\|T(\zeta)\| \leqq\|T\|
$$

and a mapping $\Phi_{\zeta}: T \rightarrow T(\zeta)$ is a $*$-homomorphism. Put $F_{\zeta}(\xi)=\Phi_{\zeta}(F(\xi))$ for $\xi \in K$. Then 


$$
F(\xi)=\int^{\oplus} F_{\zeta}(\xi) d \nu(\zeta)
$$

and $F_{\zeta}$ belongs to some skew distribution $m_{\zeta}$ on $(K, \Gamma)$. It follows that

$$
\left[F(\xi), F(\eta)^{*}\right]_{+}=\int^{\oplus} \lambda_{\zeta}(\xi, \eta) 1(\zeta) d \nu(\zeta)
$$

for some complex number $\lambda_{\zeta}(\xi, \eta) \in \mathbf{C}$. Since $K$ is separable, it contains a countable dense linear subset $K_{0}$ on $\mathbf{Q}$. Then there is a $\nu$-null set $N \subset Z$ such that

$$
\left[F_{\zeta}(\xi), F_{\zeta}(\eta)^{*}\right]_{+}=\lambda_{\zeta}(\xi, \eta) 1(\zeta)
$$

and $\left\|F_{\zeta}(\xi)\right\| \leqq\|F(\xi)\|$ for $\zeta \notin N$ and $\xi, \eta \in K_{0}$. Since

$$
\begin{gathered}
\int^{\oplus} \lambda_{\zeta}\left(\lambda \xi_{1}+\xi_{2}, \eta\right) 1(\zeta) d \nu(\zeta)=\left[F\left(\lambda \xi_{1}+\xi_{2}\right), F(\eta)^{*}\right]_{+} \\
=\lambda\left[F\left(\xi_{1}\right), F(\eta)^{*}\right]_{+}+\left[F\left(\xi_{2}\right), F(\eta)^{*}\right]_{+} \\
=\int^{\oplus}\left\{\lambda \lambda_{\zeta}\left(\xi_{1}, \eta\right)+\lambda_{\zeta}\left(\xi_{2}, \eta\right)\right\} 1(\zeta) d \nu(\zeta), \\
\int^{\oplus} \lambda_{\zeta}(\xi, \xi) 1(\zeta) d \nu(\zeta)=\left[F(\xi), F(\xi)^{*}\right]_{+} \geqq 0
\end{gathered}
$$

and

$$
\left|\lambda_{\xi}(\xi, \xi)\right| \leqq\left\|\left[F(\xi), F(\xi)^{*}\right]_{+}\right\|
$$

for $\xi, \xi_{1}, \xi_{2}, \eta \in K_{0}$ and $\lambda \in \mathbf{Q}$, it follows that $\lambda_{\xi}$ is a positive definite bounded bilinear functional on $K_{0}$ as well as on $K$. Hence a bounded positive operator $t(\zeta)$ exists on $K$ for $\zeta \in Z-N$ satisfying

$$
\left[F_{\zeta}(\xi), F_{\zeta}(\eta)^{*}\right]_{+}=\lambda_{\zeta}(\xi, \eta) 1(\zeta)=2(t(\zeta) \xi \mid \eta) 1(\zeta)
$$

for $\xi, \eta \in K$. Define $t(\zeta)=0$ for $\zeta \in N$. Then the mapping $\zeta \rightarrow t(\zeta)$ is a $\nu$-measurable function on $Z$ with values in $\mathbf{B}(K)$ and

$$
\left[F(\xi), F(\eta)^{*}\right]_{+}=2 \int^{\oplus}(t(\zeta) \xi \mid \eta) 1(\zeta) d \nu(\zeta)
$$

for any $\xi, \eta \in K$. Since $E$ is a faithful normal trace with $\|E\|=1$ over 2 , there exists a $\nu$-measurable field $\zeta \rightarrow E_{\zeta}$ of finite faithful normal traces on $Z$ such that

$$
E=\int^{\oplus} E_{\zeta} d \nu(\zeta)
$$


It follows that

$$
2\left(\iota \xi \mid \eta ;=E\left(\left[F(\xi), F(\eta)^{*^{-}}\right]_{+}\right)=2 \int\left(t\left(\zeta_{\zeta}^{\prime}\right) \xi \mid \eta\right) E_{\zeta}(1(\zeta)) d \nu(\zeta)\right.
$$

and hence

$$
t=\int E_{\zeta}(1(\zeta)) t(\zeta) d \nu(\zeta)
$$

Definiticn. A distribution $m$ is a canonical skew distribution, if for $(F, \mathfrak{Y}) \in m$

$$
\left[F(\xi), F(\eta)^{*}\right]_{+}=(\xi \mid \eta) 1
$$

Remark 1. If $m$ is a factor skew distribution with the covariance operator $t$, then

$$
\left[F(\xi), F(\eta)^{*}\right]_{\uparrow}=2(\iota \xi \mid \eta) 1 .
$$

Since $t=\Gamma t \Gamma, K$ is a pre-Hilbert space with respect to the inner product $(\mid)_{t}$ defined by

$$
(\xi \mid \eta)_{t}=(2 t \xi \mid \eta)
$$

for $\xi, \eta \in K$. Denote by $K_{t}$ the completion of $K$ with respect to $(\mid)_{t}$. Since $\Gamma$ can be extended to $K_{t}$, we shall denote it by the same letter $\Gamma$. Then

$$
\left[F(\xi), F(\eta)^{*}\right]_{+}=(\xi ; \eta)_{t} 1
$$

that is, $F$ is a canonical skew distribution on $\left(K_{t}, \Gamma\right)$, which generates a self dual CAR algebra $\mathfrak{2}_{S D C}\left(K_{t}, \Gamma\right)$. Choosing a standard representative $\left(F, \mathfrak{Y}\right.$.), we know that $\mathfrak{?}$ is a hyperfinite $I I_{1}$ factor, if $K$ is of infinite dimension.

If the underlying Hilbert space $\mathfrak{C}$ of a von Neumann algebra $\mathfrak{U}$ is separable, then 2 ? has a countable generator $\mathfrak{M}$. Let $\mathfrak{B}_{0}$ and $\mathfrak{B}$ denote *-algebras algebraically generated by $\mathfrak{M}$ on $\mathbf{Q}$ and $\mathbf{C}$ respectively. Then $\mathfrak{B}_{0}$ is countable and the unit ball of $\mathfrak{B}_{0}$ is uniformly dense in the unit ball of $\mathfrak{B}$. Since $\mathfrak{B}$ is strongly dense in $\mathfrak{A}$., the unit ball of $\mathfrak{B}$ is strongly dense in the unit ball $\mathfrak{2}_{\cdot}$ of $\mathfrak{A}$ by the Kaplansky's density theorem and 
hence ${ }^{2} \cdot{ }_{1}$ is separable. Since the unit ball of a countably decomposable von Neumann algebra is metrizable by the strong topology, $\mathfrak{N} \cdot 1$ satisfies the second axiom of countability. Thus any subset $\mathfrak{N}$ of $\mathfrak{L}_{1}$ contains a coutable subset $\mathfrak{N}_{0}$ which is strongly dence in $\mathfrak{N}$.

Utilizing the same notations as in the last lemma, we have the following

Lemma 3. A necessary and sufficient condition that a skew distribution $m$ be $\mathscr{U}$-invariant is that there be $(F, \mathfrak{Y} \cdot) \in m$ and $t(\zeta)$ as in Lemma 2 such that

(i) $\quad E\left(F\left(U \xi_{1}\right) \ldots F\left(U \xi_{n}\right)\right)=E\left(F\left(\xi_{1}\right) \ldots F\left(\xi_{n}\right)\right)$ for every $U \in \mathscr{U}$; and

(ii) $t(\zeta)$ is a scalar operator $\nu$-almost everywhere.

Proof. Necessity: By virtue of Lemma 2, there exists a representative $F \in m$ such that

$$
\begin{aligned}
& 2 \int^{\oplus}\left(t(\zeta) U \xi \mid U_{\eta}\right) 1(\zeta) d \nu(\zeta)=\left[F(U \xi), F(U \eta)^{*}\right]_{+} \\
& \quad=\left[F(\xi), F(\eta)^{*}\right]_{+}=2 \int^{\oplus}(t(\zeta) \xi \mid \eta) 1(\zeta) d \nu(\zeta) .
\end{aligned}
$$

Since $\mathfrak{S}$ is separable, $\mathscr{U}$ contains a countable family $\mathscr{U}_{0}$ which is strongly dense in $\mathscr{U}$ by the preceding discussion. Thus there is a $\nu$-null set $N \subset Z$ such that

$$
(t(\zeta) U \xi \mid U \eta)=(t(\zeta) \xi \mid \eta)
$$

for any $\zeta \in Z-N, U \in \mathscr{U}_{0}$ and $\xi, \eta \in K_{0}$. Since $\left(\mathscr{U}_{0}\right)^{\prime}=\mathscr{U}^{\prime}$ is the algebra of scalar operators, it follows that $t(\zeta)$ is a scalar operator for $\zeta \in Z-N$.

Sufficiency: If $t(\zeta)$ is a scalar operator $\nu$-almost everywhere, then

$$
\begin{gathered}
{\left[F(U \xi), F(U \eta)^{*}\right]_{+}=2 \int^{\oplus}(t(\zeta) U \xi \mid U \eta) 1(\zeta) d \nu(\zeta)} \\
\quad=2 \int^{\oplus}(t(\zeta) \xi \mid \eta) 1(\zeta) d \nu(\zeta)=\left[F(\xi), F(\eta)^{*}\right]_{\perp} .
\end{gathered}
$$

Further, since $F(U \xi), U \in \mathscr{U}$ is strongly continuous, faithful linear mapping in $\xi$ with $F(U \Gamma \xi)=F(U \xi)^{*}, U$ induces an automorphism $\tau(U)$ of $\mathfrak{2}\left(K_{0}\right)$ 
for any finite $K_{0}$ in $K$ such that $\tau(U) F(\xi)=F(U \xi), \xi \in K_{0}$ and $E(\tau(U) A)$ $=E(A), A \in \mathfrak{R}\left(K_{0}\right)$ by (i).

Lemma 4. Let $B$ be a canonical skew distribution. If $\left[\xi_{i}, \Gamma \xi_{i}\right.$ : $i=1, \ldots, n]$ and $\left[\eta_{j}, \Gamma \eta_{j}: j=1, \ldots, m\right]$ are orthogonal, then the quasifree state $E$ with $E\left(B(\eta)^{*} B(\xi)\right)=2^{-1}(\xi \mid \eta)$ on a $C^{*}$-algebra of a self dual CAR algebra $\mathfrak{H}_{S D C}(K, \Gamma)$ satisfies

$$
E\left(B\left(\xi_{1}\right) \ldots B\left(\xi_{n}\right) B\left(\eta_{1}\right) \ldots B\left(\eta_{m}\right)\right)=E\left(B\left(\xi_{1}\right) \ldots B\left(\xi_{n}\right)\right) E\left(B\left(\eta_{1}\right) \ldots B\left(\eta_{m}\right)\right),
$$

where $\left[\omega_{k}: k=1, \ldots, l\right]$ denotes the subspace spanned by $\omega_{1}, \ldots, \omega_{l}$.

Proof. If $n$ or $m$ are odd, then the left side and at least one of the factors in the right side are 0 due to $E\left(B\left(\xi_{i}\right) B\left(\eta_{j}\right)\right)=E\left(B\left(\eta_{j}\right) B\left(\xi_{i}\right)\right)=0$. If $n$ and $m$ are even,

$$
E\left(B\left(\xi_{1}\right) \ldots B\left(\xi_{n}\right) B\left(\eta_{1}\right) \ldots B\left(\eta_{m}\right)\right)=\sum_{\sigma} \operatorname{sgn}(\sigma) \prod_{j=1}^{k} E\left(B\left(\xi_{\sigma(2 j-1)}\right) B\left(\xi_{\sigma(2 j)}\right)\right)
$$

where $2 k=n+m, \xi_{n+i}=\eta_{i}$ for $i=1, \ldots, m$, sgn is the signature of the permutation $\sigma$ satisfying

$$
\sigma(1)<\sigma(3)<\cdots<\sigma(2 k-1) \text { and } \sigma(2 j-1)<\sigma(2 j)
$$

for $j=1, \ldots, k$. If there is $j$ with $1 \leqq j \leqq k$ such that $1 \leqq \sigma(2 j-1) \leqq n$ and $n+1 \leqq \sigma(2 j) \leqq n+m$, then

$$
\prod_{j=1}^{k} E\left(B\left(\xi_{\sigma(2 j-1)}\right) B\left(\xi_{\sigma(2 j)}\right)\right)=0 .
$$

Therefore we have only to consider the sum over all permutations $\sigma$ satisfying

$$
1 \leqq \sigma(2 j-1)<\sigma(2 j) \leqq n
$$

for $j=1, \ldots, 2^{-1} n$ and

$$
n+1 \leqq \sigma(2 i-1+n)<\sigma(2 i+n) \leqq n+m
$$

for $i=1, \ldots, 2^{-1} m$. Let $s$ and $t$ denote the permutations

$$
s=\left(\begin{array}{ccc}
1 & 2 \ldots & n \\
\sigma(1) \sigma(2) \ldots \sigma(n)
\end{array}\right) \text { and } t=\left(\begin{array}{ccc}
n+1 & n+2 \ldots & n+m \\
\sigma(n+1) \sigma(n+2) \ldots \sigma(n+m)
\end{array}\right)
$$


Then $\operatorname{sgn}(\sigma)=\operatorname{sgn}(s) \operatorname{sgn}(t)$ for $\sigma$ considered. Consequently

$$
\begin{aligned}
& E\left(B\left(\xi_{1}\right) \ldots B\left(\xi_{n}\right) B\left(\eta_{1}\right) \ldots B\left(\eta_{m}\right)\right) \\
& =\sum_{\sigma} \operatorname{sgn}(\sigma)\left\{\prod_{j=1}^{n / 2} E\left(B\left(\xi_{\sigma(2 j-1)}\right) B\left(\xi_{\sigma(2 j)}\right)\right)\right\}\left\{\prod_{i=1}^{m / 2} E\left(B\left(\xi_{\sigma(2 i-1+n)}\right) B\left(\xi_{\sigma(2 i+n)}\right)\right\}\right. \\
& =\left\{\sum_{s} \operatorname{sgn}(s) \prod_{j=1}^{n / 2} E\left(B\left(\xi_{s(2 j-1)}\right) B\left(\xi_{s(2 j)}\right)\right)\right\} \\
& \qquad\left\{\sum_{t} \operatorname{sgn}(t) \prod_{i=1} E\left(B\left(\eta_{t(2 i-1)}\right) B\left(\eta_{t(2 i)}\right)\right)\right\} \\
& =E\left(B\left(\xi_{1}\right) \ldots B\left(\xi_{n}\right)\right) E\left(B\left(\eta_{1}\right) \ldots B\left(\eta_{m}\right)\right),
\end{aligned}
$$

where $s$ and $t$ satisfy the condition in the definition of a quasifree state.

Since $E$ is faithful and $\nu$ has the carrier $Z$, it follows that a function $\zeta \rightarrow E_{\zeta}(1(\zeta))$ is $\nu$-measurable and $0<E_{\zeta}(1(\zeta))<+\infty \nu$-almost everywhere. Define a probability measure $\mu$ on $Z$ by $d \mu(\zeta)=E_{\zeta}(1(\zeta)) d \nu(\zeta)$. Then $\mu$ and $\nu$ are equivalent and hence the $\mu$-measurability and the $\nu$ measurability coincide.

Theorem. Let $m$ be a skew distribution on $(K, \Gamma)$ whose dimension is even or infinite. Any two of the following conditions imply the remaining one:

(i) $m$ is a factor distribution;

(ii) $m$ is $\mathscr{U}$-invariant; and

(iii) any pair of $\Gamma$-invariant orthogonal subspaces are independent with respect to $m$.

In this case $m$ is the canonical skew distribution up to a scalar constant.

Proof. (i) and (ii) imply (iii): According to (i), (ii) and Lemma 3, the covariance operator $t$ of $m$ is a scalar operator, say $2 t=\lambda 1$ for $\lambda>0$. Put $B(\xi)=\lambda^{-1 / 2} F(\xi)$. Again by (i)

$$
\left[B(\xi), B(\eta)^{*}\right]_{+}=E\left(\left[B(\xi), B(\eta)^{*}\right]_{+}\right)_{1}=\lambda^{+} E\left(\left[F(\xi), F(\eta)^{*}\right]_{+}\right)_{1}=(\xi \mid \eta)_{1},
$$

hence $B$ is a canonical skew distribution. Then $B(\xi), \xi \in K$ generate a self dual CAR algebra $\mathfrak{2}_{S D C}(K, \Gamma)$, we have 


$$
\begin{aligned}
E\left(F\left(\xi_{1}\right) \ldots F\left(\xi_{n}\right) F\left(\eta_{1}\right) \ldots F\left(\eta_{m}\right)\right) \\
=\lambda^{(n+m) / 2} E\left(B\left(\xi_{1}\right) \ldots B\left(\xi_{n}\right) B\left(\eta_{1}\right) \ldots B\left(\eta_{m}\right)\right) \\
=\lambda^{(n+m) / 2} E\left(B\left(\xi_{1}\right) \ldots B\left(\xi_{n}\right)\right) E\left(B\left(\eta_{1}\right) \ldots B\left(\eta_{m}\right)\right) \\
=E\left(F\left(\xi_{1}\right) \ldots F\left(\xi_{n}\right)\right) E\left(F\left(\eta_{1}\right) \ldots F\left(\eta_{m}\right)\right)
\end{aligned}
$$

for any orthogonal subspaces $\left[\xi_{i}, \Gamma \xi_{i}: i=1, \ldots, n\right]$ and $\left[\eta_{j}, \Gamma \eta_{j}: j=1, \ldots\right.$, $m]$ by Lemma 4 .

(ii) and (iii) imply (i): Employing the same notations as in Lemma 2 , we find a $\mu$-measurable field $\zeta \rightarrow F_{\zeta}(\xi)$ of operators on $Z$ and a $\mu$-measurable operator valued function $\zeta \rightarrow t(\zeta)$ with the remaining properties in Lemma 2 such that

$$
F(\xi)=\int^{\oplus} F_{\zeta}(\xi) d \nu(\zeta)
$$

and

$$
t=\int t(\zeta) d \nu(\zeta)
$$

It follows from (ii) and Lemma 3 that $t(\zeta)$ is a scalar operator, say $2 t(\zeta)$ $=\lambda(\zeta) 1$ and $\lambda(\zeta)>0$ for $\zeta \in Z-N$ and $\mu(N)=0$. Putting $B_{\zeta}(\xi)=\lambda(\zeta)^{-1 / 2}$ $F_{\zeta}(\xi)$ for $\zeta \in Z-N$ and $B_{\zeta}(\xi)=0$ for $\zeta \in N$, we have a $\mu$-measurable field $\zeta \rightarrow B_{\zeta}(\xi)$ of canonical skew distributions. According to Lemma 4

$$
\begin{aligned}
E & \left(F\left(\xi_{1}\right) \ldots F\left(\xi_{2 n}\right) F\left(\eta_{1}\right) \ldots F\left(\eta_{2 m}\right)\right) \\
& =\int \lambda(\zeta)^{n+m} E_{\zeta}\left(B_{\zeta}\left(\xi_{1}\right) \ldots B_{\zeta}\left(\xi_{2 n}\right) B_{\zeta}\left(\eta_{1}\right) \ldots B_{\zeta}\left(\eta_{2 m}\right)\right) d \nu(\zeta) \\
& =\int \lambda(\zeta)^{n+m} E_{\zeta}\left(B\left(\xi_{1}\right) \ldots B_{\zeta}\left(\xi_{2 n}\right)\right) E_{\zeta}\left(B_{\zeta}\left(\eta_{1}\right) \ldots B_{\zeta}\left(\eta_{2 m}\right)\right) d \nu(\zeta) .
\end{aligned}
$$

On the other hand

$$
\begin{aligned}
& E\left(F\left(\xi_{1}\right) \ldots F\left(\xi_{2 n}\right)\right) E\left(F\left(\eta_{1}\right) \ldots F\left(\eta_{2 m}\right)\right) \\
& \quad=\int \lambda(\zeta)^{n} E_{\zeta}\left(B_{\zeta}\left(\xi_{1}\right) \ldots B_{\zeta}\left(\xi_{2 n}\right)\right) d \nu(\zeta) \int \lambda(\zeta)^{m} E_{\zeta}\left(B_{\zeta}\left(\eta_{1}\right) \ldots B_{\zeta}\left(\eta_{2 m}\right)\right) d \nu(\zeta) .
\end{aligned}
$$

Selecting $\xi_{i}$ and $\eta_{j}$ being mutually orthogonal such that $\xi_{n+i}=\Gamma \xi_{i}$ and $\eta_{m+j}=\Gamma \eta_{j}$. Then by (iii)

$$
\int\{\lambda(\zeta) / 2\}^{n+m} d \mu(\zeta)=\int\{\lambda(\zeta) / 2\}^{n} d \mu(\zeta) \int\{\lambda(\zeta) / 2\}^{m} d \mu(\zeta)
$$


for any integers $n \geqq 0$ and $m \geqq 0$. Therefore $\lambda(\zeta)$ is constant $\mu$-almost everywhere and hence $\lambda(\zeta)=\lambda$ for some $\lambda>0 \mu$-almost everywhere. Let $B(\xi)$ be an operator in $\mathfrak{A}$ which corresponds to $\zeta \rightarrow B_{\zeta}(\xi)$. Then

$$
F(\xi)=\int^{\oplus} F_{\zeta}(\xi) d \nu(\zeta)=\int^{\oplus} \lambda^{1 / 2} B_{\zeta}(\xi) d \nu(\zeta)=\lambda^{12} B(\xi)
$$

and hence $B$ is a canonical skew distribution on $(K, \Gamma)$. Taking a standard representative $(F, \mathfrak{N}) \in m$, we can conclude that the von Neumann algebra $\mathfrak{U}$ is a factor.

(iii) and (i) imply (ii): By (iii), if $(\xi \mid \eta)=(\xi \mid \Gamma \eta)=0$, then

$$
(t \xi \mid \eta)=E\left(m(\eta)^{*} m(\xi)\right)=E\left(m(\eta)^{*}\right) E(m(\xi))=0 .
$$

It follows from Lemma 1 that $2 t=\lambda 1$ for some $\lambda>0$. Put $B(\xi)=$ $\lambda^{-1 / 2} F(\xi)$. Then $B$ is a canonical skew distribution by (i). Since

$$
\begin{aligned}
& E\left(F\left(\xi_{1}\right) \ldots F\left(\xi_{m}\right)\right)=\lambda^{n} E\left(B\left(\xi_{1}\right) \ldots B\left(\xi_{2 n}\right)\right) \\
& \quad=\lambda^{n} \sum_{\sigma} \operatorname{sgn}(\sigma) \prod_{j=1}^{n} E\left(B\left(\xi_{\sigma(2 j-1)}\right) B\left(\xi_{\sigma(2 j)}\right)\right) \\
& =\lambda^{n} \sum_{\sigma} \operatorname{sgn}(\sigma) \prod_{j=1}^{n} E\left(B\left(U \xi_{\sigma(2 j-1)}\right) B\left(U \xi_{\sigma(2 j)}\right)\right) \\
& =\lambda^{n} E\left(B\left(U \xi_{1}\right) \ldots B\left(U \xi_{2 n}\right)\right)=E\left(F\left(U \xi_{1}\right) \ldots F\left(U \xi_{2 n}\right)\right),
\end{aligned}
$$

it follows from Lemma 3 that $m$ is $\mathscr{U}$-invariant.

Remark 2. In case where the last theorem is valid, the covariance operator of $m$ is a scalar operator. Making use of the results of Segal [5], we have that if $A \in \mathfrak{U}$ and $\tau(U) A=A$ for all $U \in \mathscr{U}$, then $A$ is a scalar operator, where $\tau(U)$ is the $*$-automorphism induced by

$$
\tau(U) F(\xi)=F(U \xi) .
$$

\section{Appendix}

Let $K$ be a complex Hilbert space and $\Gamma$ be an antiunitary involution. A projection $e$ on $K$ is called a basis projection if $\Gamma e \Gamma=1-e$. Restricting the coefficient to the real number field and define the inner product $(\mid)_{r}$ by 


$$
(\xi \mid \eta)_{r}=\mathfrak{R}(\xi \mid \eta)
$$

we have a real Hilbert space $\left(K,(\mid)_{r}\right)$, which we denote by $K_{r}$. Here $\Re$ denotes the real part. Then the operator $\mathbf{i}$ of multiplying $i$ on $K_{r}$ is an orthogonal transformation on $K_{r}$ with $\mathbf{i}^{2}=-1$. Since the set $\{\xi \in K: \Gamma \xi$ $=\xi\}$ forms a real Hilbert space for the inner product $(\mid)_{r}$, we designate it by $H$. Then $\mathbf{i} H$ is a set $\{\xi \in K: \Gamma \xi=-\xi\}$ and $K_{r}=H \oplus \mathbf{i} H$. The complexification $H+i H$ of $H$ with the inner product

$$
\left(\xi_{1}+i \xi_{2} \mid \eta_{1}+i \eta_{2}\right)=\left(\xi_{1} \mid \eta_{1}\right)-\left(\xi_{2} \mid \eta_{2}\right)+i\left\{\left(\xi_{2} \mid \eta_{1}\right)-\left(\xi_{1} \mid \eta_{2}\right)\right\}
$$

is naturally isomorphic to $K$. For any orthogonal transformation $v$ on $H$ there corresponds a Bogoliubov transformation $u$ on $K$ such that

$$
u\left(\xi_{1}+i \xi_{2}\right)=v \xi_{1}+i v \xi_{2}
$$

for $\xi_{1}, \xi_{2} \in H$. Conversely, since every Bogoliubov transformation is reduced by $H$, the restriction $v$ of $u$ onto $H$ is an orthogonal transformation on $H$. Since $v=1$ iff $u=1$, this correspondence is bijective. Let $\Lambda$ be an orthogonal transformation on $H$ with $\Lambda^{2}=-1$ and $e$ be the projection onto the complex subspace of $K$ such that

$$
e K=\left\{\xi-i \Lambda \xi:{ }_{\Perp}^{E} \xi \in H\right\} .
$$

Then $e$ is a basis projection, $\Gamma(\xi+i \Lambda \xi)=\xi-i \Lambda \xi$ and

$$
(1-e) K=\{\xi+i \Lambda \xi: \xi \in H\} .
$$

Proposition 1. There is a bijection between the family of pairs $\{e$, $1-e\}$ of basis projections on $(K, \Gamma)$ and the family of pairs $\left\{\Lambda,{ }^{t} \Lambda\right\}$ of orthogonal transformations with $\Lambda^{2}=-1$ on $H$.

Proof. With $\Lambda$ satisfying ${ }^{t} \Lambda \Lambda=\Lambda^{t} \Lambda=1$ and $\Lambda^{2}=-1$, we associate basis projections $e$ and $1-e$ as above.

Suppose that $e$ is a basis projection. Let $H$ be the real Hilbert space $\{\eta+\Gamma \eta: \eta \in e K\}$. Choose $\xi \in H$. If $\xi=\eta+\Gamma \eta$ and $\xi=\eta^{\prime}+\Gamma \eta^{\prime}$ for $\eta$ and $\eta^{\prime} \in e K$. Then $\eta-\eta^{\prime}=\Gamma\left(\eta-\eta^{\prime}\right)$ and $\eta-\eta^{\prime} \in e K$, which implies that $\eta=\eta^{\prime}$. Thus we may define a transformation $\Lambda_{0}$ on $H$ by

$$
\Lambda_{0}(\eta+\Gamma \eta)=i(\eta-\Gamma \eta)=i \eta+\Gamma(i \eta)
$$


for $\eta \in e k$. Then $\Lambda_{0}$ is an orthogonal transformation with $\Lambda_{0}^{2}=-1$. Since for $\tilde{\xi}=\eta+\Gamma \eta$ and $\eta \in e K$ we have

$$
\xi+i \Lambda_{0} \xi=2 \Gamma \eta \in(1-e) K \quad \text { and } \xi-i \Lambda_{0} \xi=2 \eta \in e K,
$$

it follows that $\Lambda_{0}$ is associated with $e$ and $1-e$. Hence the mapping $\left\{\Lambda,{ }^{t} \Lambda\right\} \rightarrow\{e, 1-e\}$ defined above is onto. Suppose now that $e$ is a basis projection associated with a given orthogonal transformation $\Lambda$ with $\Lambda^{2}=$ -1 such that

$$
e K=\{\xi-i \Lambda \xi: \xi \in H\},
$$

and that $\Lambda_{0}$ is associated with $e$ as above. Since, then, for any $\xi \in H$ we have $\eta=\xi-i \Lambda \xi \in e K$ and $\Gamma \eta=\xi+i \Lambda \xi \in(1-e) K$, it follows that

$$
\eta+\Gamma \eta=2 \xi \text { and } i(\eta-\Gamma \eta)=2 \Lambda \xi \text {. }
$$

Therefore $\Lambda_{0} \xi=\Lambda \xi$ for $\xi \in H$, that is, $\Lambda_{0}=\Lambda$.

Remark 3. It is clear that a self dual CAR algebra $\mathfrak{A}_{S D C}(K, \Gamma)$ on $(K, \Gamma)$ and a Clifford algebra $\mathfrak{2}_{C L I}(H)$ coincide, if $K$ is of even or infinite dimension.

A family $\left\{K_{\iota}: \iota \in I\right\}$ of $\Gamma$-invariant subspaces of $(K, \Gamma)$ is said to be independent with respect to a quasifree state $\varphi$ on $\overline{\mathfrak{A}}_{S D C}(K, \Gamma)$ if

$$
\varphi\left(A_{\iota_{1}} \ldots A_{\iota_{n}}\right)=\varphi\left(A_{\iota_{1}}\right) \ldots \varphi\left(A_{\iota_{n}}\right)
$$

for any $A_{\iota} \in \overline{\mathfrak{N}}_{S D C}\left(K_{\iota}, \Gamma\right)$ and for any $\iota_{1}, \ldots, \iota_{n} \in I$, where $\overline{\mathfrak{N}}_{S D C}\left(K_{\iota}, \Gamma\right)$ is the $C^{*}$-subalgebra of $\overline{\mathfrak{A}}_{S D C}(K, \Gamma)$ generated by $B(\xi), \xi \in K$ and the identity. It is shown in $[2]$ that there is a one to one correspondence between an operator $s$ on $(K, \Gamma)$ with $0 \leqq s \leqq 1$ and $\Gamma s \Gamma=1-s$, and a quasifree state $\varphi$ on $\overline{\mathfrak{A}}_{S D C}(K, \Gamma)$ as the following

$$
(s \xi \mid \eta)=\varphi\left(B(\eta)^{*} B(\xi)\right) .
$$

Proposition 2. Let $\varphi$ be a quasifree state on $\overline{\mathfrak{A}}_{S D C}(K, \Gamma)$. Then $\varphi$ is central if and only if any pair of $\Gamma$-invariant subspaces are independent with respect to $\varphi$.

Proof. The only if part is clear from Lemma 4. It suffices to show the if part. If $\xi$ and $\eta$ are non zero vectors with $(\xi \mid \eta)=(\xi \mid \Gamma \eta)$, then 


$$
(s \xi \mid \eta)=\varphi\left(B(\eta)^{*} B(\xi)\right)=\varphi\left(B(\eta)^{*}\right) \varphi(B(\xi))=0 .
$$

It follows from Lemma 1 that $s$ is a scalar operator, that is, $s=2^{-1} 1$.

\section{Acknowledgement}

The author would like to thank Professor Araki for his valuable advices and for his warm hospitality at Research Institute for Mathematical Sciences. His thanks also goes to Professor Takenouchi and $\mathrm{Mr}$. Kitagawa for their comments.

\section{References}

[1] Araki, H., On the diagonalization of a bilinear Hamiltonian by a Bogoliubov transformation, Publ. RIMS, Kyoto Univ. 4 (1968), 387-412.

[2] - - On quasifree states of CAR and Bogoliubov automorphisms, Publ. RIMS, Kyoto Univ. 6 (1970/71), 385-442.

[3] Dixmier, J., Les Algèbres d'Opérateurs dans l'Espace Hilbertien, Gauthier-Villars, Paris, 1957.

[4] Segal, I.E., Tensor algebras over Hilbert spaces, II, Ann. of Math. 63 (1956), $160-175$.

[5] Ergodic subgroups of the orthogonal group of a real Hilbert space, Ann. of Math. 66 (1957), 297-303.

[6] , Distributions in Hilbert space and canonical systems of operators, Trans. Amer. Math. Soc. 88 (1958), 12-41.

[7] Shale, D. and W.F. Stinespring, States of the Clifford algebra, Ann. of Math. 80 (1964), 365-381. 
